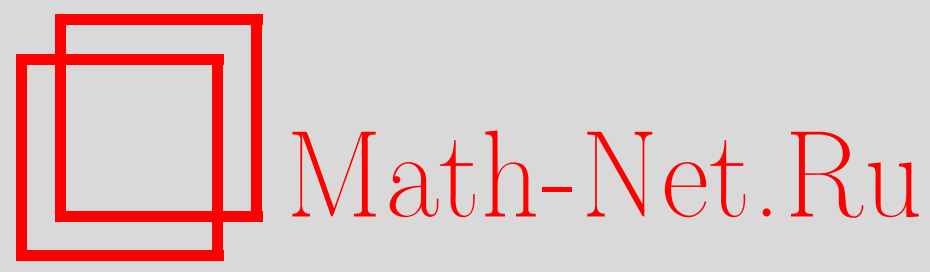

О. А. Матевосян, С. В. Пикулин, Об усреднении слабонелинейных дивергентных эллиптических операторов в перфорированном кубе, Матем. заметки, 2000, том 68, выпуск 3, 390-398

DOI: https://doi.org/10.4213/mzm956

Использование Общероссийского математического портала Math-Net.Ru подразумевает, что вы прочитали и согласны с пользовательским соглашением http://www.mathnet.ru/rus/agreement

Параметры загрузки:

IP: 54.224 .187 .69

26 апреля 2023 г., 03:41:48

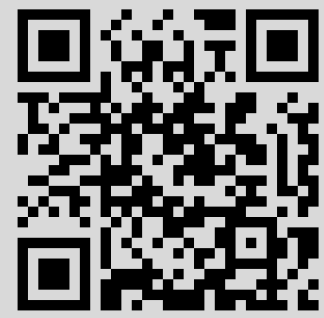




\title{
ОБ УСРЕДНЕНИИ СЛАБОНЕЛИНЕЙНЫХ ДИВЕРГЕНТНЫХ ЭЛЛИПТИЧЕСКИХ ОПЕРАТОРОВ В ПЕРФОРИРОВАННОМ КУБЕ
}

\author{
О. А. Матевосян, С.В. Пикулин
}

В настоящей работе для слабонелинейного дивергентного эллиптического уравнения второго порядка с младшим членом, растущим по неизвестной функции степенньм образом, доказывается, что последовательность решений в перфорированном кубе стремится к решению в неперфорированном кубе, если радиусы дыр стремятся к нулю со скоростью, зависящей от показателя степени в младшем члене.

Библиографория: 12 названий.

Изучению вопросов теории усреднения для перфорированных областей посвящено много монографий и работ (см. [1]-[5]), в которых на поведение решений уравнений при подходе к дыркам ставятся какие-либо ограничения, например, - граничные условия. Однако, для усреднения решений уравнений с достаточно сильным нелинейным поглощением такие ограничения не нужны. Подобная ситуация была обнаружена и в теории устранения особенностей. В связи с этим отметим работы [6]-[11].

Введем следующие обозначения:

$$
\begin{aligned}
& B\left(x_{0}, R\right)=\left\{x \in \mathbb{R}^{n}:\left|x-x_{0}\right| \leqslant R\right\} \text { - замкнутьй шар в } \mathbb{R}^{n} \text { радиуса } R \text { с центром } \\
& \text { в точке } x_{0} ; \\
& S\left(x_{0}, R\right) \equiv \partial B\left(x_{0}, R\right)=\left\{x \in \mathbb{R}^{n}:\left|x-x_{0}\right|=R\right\} \text { - сфера в } \mathbb{R}^{n} ; \\
& Q(0,1)=\left\{x \in \mathbb{R}^{n}: 0<x_{i}<1, i=1, \ldots, n\right\} \text { - открытьй куб в } \mathbb{R}^{n}, n>2 .
\end{aligned}
$$

Пусть в кубе $Q(0,1)$ лежат $m$ замкнутых шаров $B\left(y_{k, m}, R_{m}\right)$ с центрами в точках $y_{k, m}$ и одинакового радиуса $R_{m}$.

Положим $Q_{m}(0,1)=Q(0,1) \backslash \bigcup_{k=1}^{m} B\left(y_{k, m}, R_{m}\right)$.

Рассмотрим задачу

$$
\begin{aligned}
L u_{m}(x) & \equiv \sum_{i, j=1}^{n} \frac{\partial}{\partial x_{i}}\left(a_{i j}(x) \frac{\partial u_{m}(x)}{\partial x_{j}}\right)=a(x)\left|u_{m}(x)\right|^{\sigma-1} u_{m}(x), & x \in Q_{m}(0,1), \\
u_{m}(x) & =\varphi(x), & x \in \partial Q(0,1),
\end{aligned}
$$

где $a(x), a_{i j}(x)$ - ограниченные измеримые функции, $x=\left(x_{1}, \ldots, x_{n}\right), a_{i j}(x) \equiv a_{j i}(x)$, $a(x) \geqslant a_{0}>0$ и вьполняются условия:

$$
\lambda^{-1}|\xi|^{2} \leqslant \sum_{i, j=1}^{n} a_{i j}(x) \xi_{i} \xi_{j} \leqslant \lambda|\xi|^{2}, \quad \lambda=\text { const }>0
$$

для всех $\xi=\left(\xi_{1}, \ldots, \xi_{n}\right) \in \mathbb{R}^{n},|\xi|^{2}=\sum_{i=1}^{n} \xi_{i}^{2} ; a_{0}=$ const; $\sigma>n /(n-2) ; \varphi(x) \in$ $H^{1}\left(Q_{m}(0,1)\right) \cap L_{\infty}\left(Q_{m}(0,1)\right)$. 
ОПРЕДЕЛЕниЕ. Функция $u_{m}(x) \in H^{1}\left(Q_{m}(0,1)\right) \cap L_{\infty}\left(Q_{m}(0,1)\right)$ назьвается обобщенным решением задачи $(1),(2)$, если для любой функции $\psi(x) \in C_{0}^{\infty}\left(Q_{m}(0,1)\right)$ она удовлетворяет интегральному соотношению

$$
\sum_{i, j=1}^{n} \int_{Q_{m}(0,1)} a_{i j}(x) \frac{\partial u_{m}(x)}{\partial x_{j}} \frac{\partial \psi(x)}{\partial x_{i}} d x+\int_{Q_{m}(0,1)} a(x)\left|u_{m}(x)\right|^{\sigma-1} u_{m}(x) \psi(x) d x=0
$$

и при любой функции $\chi(x) \in C^{\infty}\left(Q_{m}(0,1)\right)$, равной нулю в окрестности $\bigcup_{k=1}^{m} B\left(y_{k, m}\right.$, $\left.R_{m}\right)$, справедливо включение $\chi(x)\left(u_{m}(x)-\varphi(x)\right) \in \stackrel{\circ}{H}^{1}\left(Q_{m}(0,1)\right)$.

Для задачи (1), (2) не вьполняются, вообще говоря, теоремы единственности, так как на границах дырок перфорированной области $Q_{m}(0,1)$ не заданы никакие граничные условия. Мы покажем, что при некоторых условиях предел решений задачи (1), (2) будет существовать, причем этот предел не зависит от выбора последовательности $u_{m}(x)$; здесь $u_{m}(x)$ - любое решение задачи $(1),(2)$.

Пусть $u(x)$ - решение следующей задачи:

$$
\begin{aligned}
L u(x) & =a(x)|u(x)|^{\sigma-1} u(x), & & x \in Q(0,1), \\
u(x) & =\varphi(x), & & x \in \partial Q(0,1) .
\end{aligned}
$$

TEOPEMA 1. Пyсmb

$$
R_{m}=o\left(m^{(1-\sigma) /((n-2) \sigma-n)}\right) \quad \text { npu } m \rightarrow \infty
$$

Тогда

i) решение задачи (1), (2) стремится к решению задачи (4), (5) в следующем смысле: для любого положительного постоянного $d$

$$
\sup _{x \in Q_{m, d}(0,1)}\left|u_{m}(x)-u(x)\right| \rightarrow 0 \quad \text { npu } m \rightarrow \infty
$$

$2 \partial e$

$$
Q_{m, d}(0,1)=\left\{x \in Q_{m}(0,1): \operatorname{dist}\left(x, \partial B\left(y_{k, m}, R_{m}\right)\right)>d ; k=1, \ldots, m\right\}
$$

ii) общий оббем дьрок $V\left[\bigcup_{k=1}^{m} B\left(y_{k, m}, R_{m}\right)\right] \equiv\left|\bigcup_{k=1}^{m} B\left(y_{k, m}, R_{m}\right)\right|$ стремится $\kappa$ нулю при $m \rightarrow \infty$.

СлЕДСТВИЕ. Если в перфорированном кубе $Q_{m}(0,1)$ существует множество, отделенное от всех дырок фиксированной постоянной, то на этом множестве сходимость будет равномерной. 
ДокАЗАТЕЛЬСТво ТЕОРЕмы 1. і) В работе [6] получена оценка решения уравнения (1) через расстояние до гранищы области:

$$
\left|u_{m}(x)\right|<C\left(\operatorname{dist}\left(x, \partial Q_{m}(0,1)\right)\right)^{2 /(1-\sigma)},
$$

где положительная постоянная $C$ не зависит от $x$ и $m$.

Обозначим через $\Gamma(x)$ фундаментальное решение оператора $L$, имеющее особенность в начале координат 0 и положительное в $B(0, R) \backslash\{0\}$. Известно [12], что такое решение существует и удовлетворяет неравенствам

$$
C_{1}|x|^{2-n} \leqslant \Gamma(x) \leqslant C_{2}|x|^{2-n},
$$

где положительные постоянные $C_{1}$ и $C_{2}$ зависят от $\lambda$ из условия (3).

Положим

$$
g_{m}(x)=\frac{1}{m} \sum_{k=1}^{m} \Gamma\left(x-y_{k, m}\right) .
$$

Легко заметить, что на границах шаров $B\left(y_{k}, m, 2 R_{m}\right)$, концентрических с шарами $B\left(y_{k, m}, R_{m}\right)$ и имеющих радиусы $2 R_{m}$, имеет место неравенство

$$
u_{m}(x)<g_{m}(x), \quad x \in \partial B\left(y_{k, m}, 2 R_{m}\right),
$$

так как на гранищах этих шаров

$$
u_{m}(x)<C^{\prime} R_{m}^{2 /(1-\sigma)}, \quad g_{m}(x)>C^{\prime \prime} m^{-1} R_{m}^{2-n},
$$

где $C^{\prime}, C^{\prime \prime}$ - положительные постоянные. Буквой $C$ здесь и далее будем обозначать любую положительную постоянную, не зависящую от $x$ и $m$.

Предположим, что $u_{m}(x)$ не стремится к $u(x)$ в смысле $(7)$ при $m \rightarrow \infty$. Тогда без ограничения общности можно считать, что найдется положительная постоянная $C_{0}$ такая, что для любого $m_{0} \in \mathbb{N}$ существуют $m>m_{0}$ и $x_{m} \in Q_{m, d}(0,1)$ такие, что $u_{m}\left(x_{m}\right)-u\left(x_{m}\right)>C_{0}$ (аналогично рассматривается случай $\left.u_{m}\left(x_{m}\right)-u\left(x_{m}\right)<-C_{0}\right)$. Очевидно, что $g_{m}\left(x_{m}\right)<C_{0}^{\prime} d^{2-n} m^{-1}=C_{0}^{\prime \prime} m^{-1}$, поскольку $x_{m} \in Q_{m, d}(0,1)$. Положим

$$
h_{m}(x)=\frac{C_{0}}{2} \frac{g_{m}(x)}{g_{m}\left(x_{m}\right)}, \quad x \in Q_{m}(0,1) .
$$

Тогда на границах шаров $\partial\left(\bigcup_{k=1}^{m} B\left(y_{k, m}, 2 R_{m}\right)\right)$ выполнено неравенство

$$
h_{m}(x)>\widetilde{C_{0}} R_{m}^{2-n} .
$$

Рассмотрим функцию

$$
v_{m}(x)=u_{m}(x)-u(x)-h_{m}(x), \quad v_{m}\left(x_{m}\right)>\frac{C_{0}}{2}>0 .
$$

Из (6), (8), (9) следует, что при достаточно больших $m$ и при $x \in \partial\left(Q(0,1) \backslash \bigcup_{k=1}^{m} B\left(y_{k, m}\right.\right.$, $\left.2 R_{m}\right)$ ) выполнено $v_{m}(x)<0$.

Определим множество $D \equiv\left\{x \in Q_{m}(0,1): v_{m}(x)>0\right\} \neq \varnothing$. Обозначим через $D_{0}$ компоненту связности множества $D$, содержащую точку $x_{m}$. Функция $v_{m}(x)$ достигает 
локального максимума во внутренней точке области $D_{0}$, что противоречит принципу максимума, вьполненного в силу того, что $L v_{m}(x)>0$ при $x \in D$.

Действительно, $u_{m}(x)-u(x)>h_{m}(x)>0$ при $x \in D$. Тогда

$$
\begin{aligned}
L v_{m}(x) & =L\left(u_{m}(x)-u(x)\right)-L h_{m}(x) \\
& =a(x)\left|u_{m}(x)-u(x)\right|^{\sigma-1}\left(u_{m}(x)-u(x)\right)>0 .
\end{aligned}
$$

Следовательно, наше предположение неверно, т. е. $u_{m}(x)$ стремится к $u(x)$ в смысле (7) при $m \rightarrow \infty$.

ii) Для объема шара радиуса $R$ в пространстве $\mathbb{R}^{n}$ вьполнено неравенство

$$
V[B(0, R)] \leqslant C R^{n},
$$

где $C$ зависит только от $n$. Оценим общий объем дырок:

$$
\left|\bigcup_{k=1}^{m} B\left(y_{k, m}, R_{m}\right)\right| \leqslant C m R_{m}^{n}=o\left(m^{-(2 \sigma) /((n-2) \sigma-n)}\right) \rightarrow 0 \quad \text { при } m \rightarrow \infty .
$$

Теорема 1 доказана.

ЛЕмма (обобщенное неравенство Юнга). Пусть $a, b, \varepsilon>0,0<\alpha<1$. Тогда

$$
a b \leqslant \frac{\alpha}{\varepsilon} a^{1 / \alpha}+\frac{1-\alpha}{\varepsilon^{-\alpha /(1-\alpha)}} b^{1 /(1-\alpha)} .
$$

В частности, при $\alpha=1 / 2$ выполнено $2 a b \leqslant a^{2} / \varepsilon+\varepsilon b^{2}$.

ТЕОрема 2. Пусть выполнены условия теоремы 1 и, кроме того, $R_{m}=o\left(m^{\gamma}\right)$, где $\gamma<(1-\sigma) /((n-2) \sigma-n)$. Тогда при $m \rightarrow \infty$

$$
\int_{Q(0,1) \backslash \bigcup_{k=1}^{m} B\left(y_{k, m}, 4 R_{m}\right)} \frac{\left|\nabla\left(u_{m}(x)-u(x)\right)\right|^{2}+\left|u_{m}(x)-u(x)\right|^{\sigma+1}}{1+\left|u_{m}(x)-u(x)\right|} d x \rightarrow 0 .
$$

ДокАЗАтЕЛЬСтво. Рассмотрим функции $\theta_{k, m}(x) \in C^{1}(Q(0,1))$ такие, что $0 \leqslant$ $\theta_{k, m}(x) \leqslant 1, x \in Q(0,1)$,

$$
\theta_{k, m}(x)= \begin{cases}1, & x \in Q(0,1) \backslash B\left(y_{k, m}, 4 R_{m}\right) \\ 0, & x \in B\left(y_{k, m}, 2 R_{m}\right)\end{cases}
$$

и $\left|\nabla \theta_{k, m}(x)\right|<C / R_{m}, x \in Q(0,1)$. Положим $\theta_{m}(x)=\inf _{k=1, \ldots, m} \theta_{k, m}(x)$.

Рассмотрим функцию $\psi(x)=\theta_{m}^{p}(x) \eta\left(u_{m}(x)-u(x)\right)$, где

$$
\eta(\tau)= \begin{cases}\tau, & |\tau| \leqslant 1 \\ |\tau|^{q-1} \tau, & |\tau|>1\end{cases}
$$

$q>0$ таково, что $\gamma<(1-\sigma) /((n-2) \sigma-n-2 q) ; p=p(q)>2$ таково, что

$$
p=\frac{(p-2)(\sigma+q)}{1+q} \text {. }
$$


Умножим равенство $L\left(u_{m}(x)-u(x)\right)=a(x)\left(\left|u_{m}(x)\right|^{\sigma-1} u_{m}(x)-|u(x)|^{\sigma-1} u(x)\right)$ на $\psi(x)$ и проинтегрируем по $Q(0,1) ;$ получим

$$
\begin{aligned}
& \int_{Q(0,1)}\left(L\left(u_{m}(x)-u(x)\right) \psi(x)\right) d x \\
& \quad \equiv \int_{Q(0,1)} \sum_{i, j=1}^{n} \frac{\partial}{\partial x_{i}}\left(a_{i j}(x) \frac{\partial}{\partial x_{j}}\left(u_{m}(x)-u(x)\right)\right) \psi(x) d x \\
& =\int_{Q(0,1)} a(x)\left(\left|u_{m}(x)\right|^{\sigma-1} u_{m}(x)-|u(x)|^{\sigma-1} u(x)\right) \psi(x) d x
\end{aligned}
$$

Оценим правую часть равенства $(11)$, используя неравенства $|a+b|^{\sigma} \leqslant C(\sigma)\left|a^{\sigma}+b^{\sigma}\right|$, $|a-b|^{\sigma} \leqslant\left|a^{\sigma}-b^{\sigma}\right|$, где $a, b>0, C(\sigma)=2^{\sigma-1}$, и при $C_{3}=\min (1 / C(\sigma), 1)=1 / 2^{\sigma-1}$, $\pm a^{\sigma}=\left|u_{m}(x)\right|^{\sigma-1} u_{m}(x), \pm b^{\sigma}=|u(x)|^{\sigma-1} u(x)$ получим

$$
\begin{aligned}
& \int_{Q(0,1)} a(x)\left(\left|u_{m}(x)\right|^{\sigma-1} u_{m}(x)-|u(x)|^{\sigma-1} u(x)\right) \psi(x) d x \\
& =\int_{\left\{\left|u_{m}(x)-u(x)\right| \leqslant 1\right\}} a(x)\left(\left|u_{m}(x)\right|^{\sigma-1} u_{m}(x)-|u(x)|^{\sigma-1} u(x)\right) \\
& \quad \times\left(u_{m}(x)-u(x)\right) \theta_{m}^{p}(x) d x \\
& \quad+\int_{\left\{\left|u_{m}(x)-u(x)\right|>1\right\}} a(x)\left(\left|u_{m}(x)\right|^{\sigma-1} u_{m}(x)-u(x)^{\sigma-1} u(x)\right) \\
& \quad \times\left|u_{m}(x)-u(x)\right|^{q-1}\left(u_{m}(x)-u(x)\right) \theta_{m}^{p}(x) d x \\
& \geqslant a_{0} C_{3} \int_{\left\{\left|u_{m}(x)-u(x)\right| \leqslant 1\right\}}\left|u_{m}(x)-u(x)\right|^{\sigma+1} \theta_{m}^{p}(x) d x \\
& \quad+a_{0} C_{3} \int_{\left\{\left|u_{m}(x)-u(x)\right|>1\right\}}\left|u_{m}(x)-u(x)\right|^{\sigma+q} \theta_{m}^{p}(x) d x .
\end{aligned}
$$

После тождественных преобразований и интегрирования по частям в левой части равенства (11) получим

$$
\begin{aligned}
& \int_{Q(0,1)} \sum_{i, j=1}^{n} \frac{\partial}{\partial x_{i}}\left(a_{i j}(x) \frac{\partial}{\partial x_{j}}\left(u_{m}(x)-u(x)\right)\right) \psi(x) d x \\
& =\int_{\left\{\left|u_{m}(x)-u(x)\right| \leqslant 1\right\}} \sum_{i, j=1}^{n} \frac{\partial}{\partial x_{i}}\left(a_{i j}(x) \frac{\partial}{\partial x_{j}}\left(u_{m}(x)-u(x)\right)\right) \\
& \quad \times\left(u_{m}(x)-u(x)\right) \theta_{m}^{p}(x) d x \\
& +\int_{\left\{\left|u_{m}(x)-u(x)\right|>1\right\}} \sum_{i, j=1}^{n} \frac{\partial}{\partial x_{i}}\left(a_{i j}(x) \frac{\partial}{\partial x_{j}}\left(u_{m}(x)-u(x)\right)\right) \\
& \quad \times\left|u_{m}(x)-u(x)\right|^{q-1}\left(u_{m}(x)-u(x)\right) \theta_{m}^{p}(x) d x
\end{aligned}
$$




$$
\begin{aligned}
& =\int_{\left\{\left|u_{m}(x)-u(x)\right| \leqslant 1\right\}}-\sum_{i, j=1}^{n} a_{i j}(x) \frac{\partial}{\partial x_{j}}\left(u_{m}(x)-u(x)\right) \frac{\partial}{\partial x_{i}}\left(u_{m}(x)-u(x)\right) \theta_{m}^{p}(x) d x \\
& +\int_{\left\{\left|u_{m}(x)-u(x)\right| \leqslant 1\right\}}-\sum_{i, j=1}^{n} a_{i j}(x) \frac{\partial}{\partial x_{j}}\left(u_{m}(x)-u(x)\right)\left(u_{m}(x)-u(x)\right) \\
& \quad \times p \theta_{m}^{p-1}(x) \frac{\partial}{\partial x_{i}} \theta_{m}(x) d x+\int_{\left\{\left|u_{m}(x)-u(x)\right|>1\right\}}-\sum_{i, j=1}^{n} a_{i j}(x) \frac{\partial}{\partial x_{j}}\left(u_{m}(x)-u(x)\right) \\
& \quad \times q\left|u_{m}(x)-u(x)\right|^{q-1} \frac{\partial}{\partial x_{i}}\left(u_{m}(x)-u(x)\right) \theta_{m}^{p}(x) d x \\
& +\int_{\left\{\left|u_{m}(x)-u(x)\right|>1\right\}}-\sum_{i, j=1}^{n} a_{i j}(x) \frac{\partial}{\partial x_{j}}\left(u_{m}(x)-u(x)\right) \\
& \quad \times\left|u_{m}(x)-u(x)\right|^{q-1}\left(u_{m}(x)-u(x)\right) p \theta_{m}^{p-1}(x) \frac{\partial}{\partial x_{i}} \theta_{m}(x) d x .
\end{aligned}
$$

Оценим первое и третье слагаемые правой части тождества (13), учитьвая (3); соответственно получим

$$
\begin{aligned}
& \int_{\left\{\left|u_{m}(x)-u(x)\right| \leqslant 1\right\}}-\sum_{i, j=1}^{n} a_{i j}(x) \frac{\partial}{\partial x_{j}}\left(u_{m}(x)-u(x)\right) \frac{\partial}{\partial x_{i}}\left(u_{m}(x)-u(x)\right) \theta_{m}^{p}(x) d x \\
& \leqslant-\frac{1}{\lambda} \int_{\left\{\left|u_{m}(x)-u(x)\right| \leqslant 1\right\}}\left|\nabla\left(u_{m}(x)-u(x)\right)\right|^{2} \theta_{m}^{p}(x) d x \\
& \int_{\left\{\left|u_{m}(x)-u(x)\right|>1\right\}}-\sum_{i, j=1}^{n} a_{i j}(x) \frac{\partial}{\partial x_{j}}\left(u_{m}(x)-u(x)\right) q\left|u_{m}(x)-u(x)\right|^{q-1} \\
& \quad \times \frac{\partial}{\partial x_{i}}\left(u_{m}(x)-u(x)\right) \theta_{m}^{p}(x) d x \\
& \leqslant-\frac{q}{\lambda} \int_{\left\{\left|u_{m}(x)-u(x)\right|>1\right\}}\left|\nabla\left(u_{m}(x)-u(x)\right)\right|^{2}\left|u_{m}(x)-u(x)\right|^{q-1} \theta_{m}^{p}(x) d x .
\end{aligned}
$$

Для оценки второго и четвертого слагаемых правой части тождества (13) используется неравенство Юнга при $\alpha=1 / 2, a=\left|\nabla\left(u_{m}(x)-u(x)\right)\right|, b=\left|\nabla \theta_{m}(x)\right|$; выбирая $\varepsilon=p \lambda^{2} /\left(\theta_{m}(x)\right)$ и $\varepsilon=\left(p \lambda^{2}\left|u_{m}(x)-u(x)\right|\right) /\left(q \theta_{m}(x)\right)$ при каждом $x$, соответственно получим

$$
\begin{aligned}
& \int_{\left\{\left|u_{m}(x)-u(x)\right| \leqslant 1\right\}}-\sum_{i, j=1}^{n} a_{i j}(x) \frac{\partial}{\partial x_{j}}\left(u_{m}(x)-u(x)\right)\left(u_{m}(x)-u(x)\right) \\
& \quad \times p \theta_{m}^{p-1}(x) \frac{\partial}{\partial x_{i}} \theta_{m}(x) d x \\
& \leqslant p \lambda \int_{\left\{\left|u_{m}(x)-u(x)\right| \leqslant 1\right\}}\left|\nabla\left(u_{m}(x)-u(x)\right)\right|\left|\nabla \theta_{m}(x)\right| \theta_{m}^{p-1}(x) d x \\
& \leqslant p \lambda \int_{\left\{\left|u_{m}(x)-u(x)\right| \leqslant 1\right\}} \theta_{m}^{p-1}(x)\left(\frac{\theta_{m}(x)}{2 p \lambda^{2}}\left|\nabla\left(u_{m}(x)-u(x)\right)\right|^{2}\right. \\
& \left.+\frac{p \lambda^{2}}{2 \theta_{m}(x)}+\left|\nabla \theta_{m}(x)\right|^{2}\right) d x
\end{aligned}
$$




$$
\begin{aligned}
&= \frac{1}{2 \lambda} \int_{\left\{\left|u_{m}(x)-u(x)\right| \leqslant 1\right\}}\left|\nabla\left(u_{m}(x)-u(x)\right)\right|^{2} \theta_{m}^{p}(x) d x \\
&+C_{4} \int_{\left\{\left|u_{m}(x)-u(x)\right| \leqslant 1\right\}}\left|\nabla \theta_{m}(x)\right|^{2} \theta_{m}^{p-2}(x) d x \\
& \int_{\left\{\left|u_{m}(x)-u(x)\right|>1\right\}}-\sum_{i, j=1}^{n} a_{i j}(x) \frac{\partial}{\partial x_{j}}\left(u_{m}(x)-u(x)\right)\left|u_{m}(x)-u(x)\right|^{q-1} \\
& \quad \times\left(u_{m}(x)-u(x)\right) p \theta_{m}^{p-1}(x) \frac{\partial}{\partial x_{i}} \theta_{m}(x) d x \\
& \leqslant p \lambda \int_{\left\{\left|u_{m}(x)-u(x)\right|>1\right\}}\left|\nabla\left(u_{m}(x)-u(x)\right)\right|\left|\nabla \theta_{m}(x)\right|\left|u_{m}(x)-u(x)\right|^{q} \theta_{m}^{p-1}(x) d x \\
& \leqslant p \lambda \int_{\left\{\left|u_{m}(x)-u(x)\right|>1\right\}} \theta_{m}^{p-1}(x)\left|u_{m}(x)-u(x)\right|^{q}\left(\frac{q \theta_{m}(x)}{2 p \lambda^{2}\left|u_{m}(x)-u(x)\right|}\right. \\
&\left.\times\left|\nabla\left(u_{m}(x)-u(x)\right)\right|^{2}+\frac{p \lambda^{2}\left|u_{m}(x)-u(x)\right|}{2 q \theta_{m}(x)}\left|\nabla \theta_{m}(x)\right|^{2}\right) d x \\
&= \frac{q}{2 \lambda} \int_{\left\{\left|u_{m}(x)-u(x)\right|>1\right\}}\left|\nabla\left(u_{m}(x)-u(x)\right)\right|^{2}\left|u_{m}(x)-u(x)\right|^{q-1} \theta_{m}^{p}(x) d x \\
&+C_{5} \int_{\left\{\left|u_{m}(x)-u(x)\right|>1\right\}}\left|\nabla \theta_{m}(x)\right|^{2}\left|u_{m}(x)-u(x)\right|^{q+1} \theta_{m}^{p-2}(x) d x
\end{aligned}
$$

Теперь оценим второе слагаемое правой части неравенства (17), полагая в неравенстве Юнга $a=\left|u_{m}(x)-u(x)\right|^{q+1} \theta_{m}^{p-2}(x), b=\left|\nabla \theta_{m}(x)\right|^{2}$ при $\alpha=(1+q) /(\sigma+q)$ и выбрав $\varepsilon=2 C_{5} \alpha /\left(a_{0} C_{3}\right)$; с учетом (10) получим

$$
\begin{aligned}
& C_{5} \int_{\left\{\left|u_{m}(x)-u(x)\right|>1\right\}}\left|\nabla \theta_{m}(x)\right|^{2}\left|u_{m}(x)-u(x)\right|^{q+1} \theta_{m}^{p-2}(x) d x \\
& \leqslant \frac{a_{0} C_{3}}{2} \int_{\left\{\left|u_{m}(x)-u(x)\right|>1\right\}}\left|u_{m}(x)-u(x)\right|^{\sigma+q} \theta_{m}^{p}(x) d x \\
& \quad+C_{6} \int_{\left\{\left|u_{m}(x)-u(x)\right|>1\right\}}\left|\nabla \theta_{m}(x)\right|^{2(\sigma+q) /(\sigma-1)} d x .
\end{aligned}
$$

Учитьвая равенства (11), (13) и оценки (14)-(18), имеем

$$
\begin{aligned}
& \int_{Q(0,1)} L\left(u_{m}(x)-u(x)\right) \psi(x) d x \\
& \leqslant-\frac{1}{2 \lambda} \int_{\left\{\left|u_{m}(x)-u(x)\right| \leqslant 1\right\}}\left|\nabla\left(u_{m}(x)-u(x)\right)\right|^{2} \theta_{m}^{p}(x) d x \\
& \quad-\frac{q}{2 \lambda} \int_{\left\{\left|u_{m}(x)-u(x)\right|>1\right\}}\left|\nabla\left(u_{m}(x)-u(x)\right)\right|^{2}\left|u_{m}(x)-u(x)\right|^{q-1} \theta_{m}^{p}(x) d x \\
& \quad+C_{4} \int_{\left\{\left|u_{m}(x)-u(x)\right| \leqslant 1\right\}}\left|\nabla \theta_{m}(x)\right|^{2} \theta_{m}^{p-2}(x) d x \\
& +\frac{a_{0} C_{3}}{2} \int_{\left\{\left|u_{m}(x)-u(x)\right|>1\right\}}\left|u_{m}(x)-u(x)\right|^{\sigma+q} \theta_{m}^{p}(x) d x \\
& +C_{6} \int_{\left\{\left|u_{m}(x)-u(x)\right|>1\right\}}\left|\nabla \theta_{m}(x)\right|^{2(\sigma+q) /(\sigma-1)} d x .
\end{aligned}
$$


Объединяя (11), (12) и (19), получим

$$
\begin{aligned}
-\frac{1}{2 \lambda} & \int_{\left\{\left|u_{m}(x)-u(x)\right| \leqslant 1\right\}}\left|\nabla\left(u_{m}(x)-u(x)\right)\right|^{2} \theta_{m}^{p}(x) d x \\
& -\frac{q}{2 \lambda} \int_{\left\{\left|u_{m}(x)-u(x)\right|>1\right\}}\left|\nabla\left(u_{m}(x)-u(x)\right)\right|^{2}\left|u_{m}(x)-u(x)\right|^{q-1} \theta_{m}^{p}(x) d x \\
& +C_{4} \int_{\left\{\left|u_{m}(x)-u(x)\right| \leqslant 1\right\}}\left|\nabla \theta_{m}(x)\right|^{2} \theta_{m}^{p-2}(x) d x \\
& +\frac{a_{0} C_{3}}{2} \int_{\left\{\left|u_{m}(x)-u(x)\right|>1\right\}}\left|u_{m}(x)-u(x)\right|^{\sigma+q} \theta_{m}^{p}(x) d x \\
& +C_{6} \int_{\left\{\left|u_{m}(x)-u(x)\right|>1\right\}}\left|\nabla \theta_{m}(x)\right|^{2(\sigma+q) /(\sigma-1)} d x \\
\geqslant & a_{0} C_{3} \int_{\left\{\left|u_{m}(x)-u(x)\right| \leqslant 1\right\}}\left|u_{m}(x)-u(x)\right|^{\sigma+1} \theta_{m}^{p}(x) d x \\
& +a_{0} C_{3} \int_{\left\{\left|u_{m}(x)-u(x)\right|>1\right\}}\left|u_{m}(x)-u(x)\right|^{\sigma+q} \theta_{m}^{p}(x) d x ;
\end{aligned}
$$

После элементарных преобразований получаем

$$
\begin{aligned}
& C_{4} \int_{\left\{\left|u_{m}(x)-u(x)\right| \leqslant 1\right\}}\left|\nabla \theta_{m}(x)\right|^{2} \theta_{m}^{p-2}(x) d x \\
& \quad+C_{6} \int_{\left\{\left|u_{m}(x)-u(x)\right|>1\right\}}\left|\nabla \theta_{m}(x)\right|^{2(\sigma+q) /(\sigma-1)} d x \\
& \geqslant a_{0} C_{3} \int_{\left\{\left|u_{m}(x)-u(x)\right| \leqslant 1\right\}}\left|u_{m}(x)-u(x)\right|^{\sigma+1} \theta_{m}^{p}(x) d x \\
&+\frac{a_{0} C_{3}}{2} \int_{\left\{\left|u_{m}(x)-u(x)\right|>1\right\}}\left|u_{m}(x)-u(x)\right|^{\sigma+q} \theta_{m}^{p}(x) d x \\
&+\frac{1}{2 \lambda} \int_{\left\{\left|u_{m}(x)-u(x)\right| \leqslant 1\right\}}\left|\nabla\left(u_{m}(x)-u(x)\right)\right|^{2} \theta_{m}^{p}(x) d x \\
&+\frac{q}{2 \lambda} \int_{\left\{\left|u_{m}(x)-u(x)\right|>1\right\}}\left|\nabla\left(u_{m}(x)-u(x)\right)\right|^{2}\left|u_{m}(x)-u(x)\right|^{q-1} \theta_{m}^{p}(x) d x \\
& \geqslant C_{7} \int_{Q(0,1)} \frac{\left|u_{m}(x)-u(x)\right|^{\sigma+1}}{1+\left|u_{m}(x)-u(x)\right|} \theta_{m}^{p}(x) d x \\
&+C_{8} \int_{Q(0,1)} \frac{\left|\nabla\left(u_{m}(x)-u(x)\right)\right|^{2}}{1+\left|u_{m}(x)-u(x)\right|} \theta_{m}^{p}(x) d x \\
& \geqslant C_{9} \int_{Q(0,1) \backslash \cup_{k=1}^{m} B\left(y_{k, m}, 4 R_{m}\right)} \frac{\left|\nabla\left(u_{m}(x)-u(x)\right)\right|^{2}+\left|u_{m}(x)-u(x)\right|^{\sigma+1}}{1+\left|u_{m}(x)-u(x)\right|} d x .
\end{aligned}
$$

Теперь докажем, что

$$
\int_{\left\{\left|u_{m}(x)-u(x)\right| \leqslant 1\right\}}\left|\nabla \theta_{m}(x)\right|^{2} \theta_{m}^{p-2}(x) d x \rightarrow 0 \quad \text { при } m \rightarrow \infty
$$


и

$$
\int_{\left\{\left|u_{m}(x)-u(x)\right|>1\right\}}\left|\nabla \theta_{m}(x)\right|^{2(\sigma+q) /(\sigma-1)} d x \rightarrow 0 \quad \text { при } m \rightarrow \infty .
$$

Действительно,

$$
\begin{aligned}
& \int_{\left\{\left|u_{m}(x)-u(x)\right| \leqslant 1\right\}}\left|\nabla \theta_{m}(x)\right|^{2} \theta_{m}^{p-2}(x) d x \leqslant \int_{Q(0,1)}\left|\nabla \theta_{m}(x)\right|^{2} d x \\
& \leqslant C_{10} m R_{m}^{n}\left(\frac{1}{R_{m}}\right)^{2}=o\left(m^{1+((n-2)(1-\sigma)) /((n-2) \sigma-n)}\right) \\
& \quad=o\left(m^{-2 /((n-2) \sigma-n)}\right) \rightarrow 0, \quad m \rightarrow \infty ; \\
& \int_{\left\{\left|u_{m}(x)-u(x)\right|>1\right\}}\left|\nabla \theta_{m}(x)\right|^{2(\sigma+q) /(\sigma-1)} d x \leqslant \int_{Q(0,1)}\left|\nabla \theta_{m}(x)\right|^{2(\sigma+q) /(\sigma-1)} d x \\
& \quad \leqslant C_{11} m R_{m}^{n}\left(\frac{1}{R_{m}}\right)^{2(\sigma+q) /(\sigma-1)}=C_{11} m R_{m}^{((n-2) \sigma-n-2 q) /(\sigma-1)} \\
& \quad=o\left(m^{1+((n-2) \sigma-n-2 q) /(\sigma-1) \cdot(1-\sigma) /((n-2) \sigma-n-2 q)}\right)=o(1) \rightarrow 0, \quad m \rightarrow \infty .
\end{aligned}
$$

Из (20), (21) и (22) следует справедливость теоремы 2.

Авторы выражают благодарность проф. В. А. Кондратьеву и доценту А. А. Конькову за полезные обсуждения.

\section{СПИСОК ЦИТИРОВАННОЙ ЛИТЕРАТУРЫ}

[1] Марченко В.А., Хруслов Е. Я. Краевые задачи в областях с мелкозернистой границей. Киев: Наукова Думка, 1974.

[2] Bensoussan A., Lions J.-L., Papanicolaou G. Asymtotic Analysis for Periodic Structures. Amsterdam: North-Holland, 1978.

[3] Damlamian A., Li Ta-Tsien. Boundary homogenization for elliptic problems // J. Math. Pures et Appl. 1987. V. 66. P. 351-361.

[4] ЖКиков В. В., Козлов С. М., Олейник О. А. Усреднение дифференциальных операторов. М.: Физматлит, 1993.

[5] Скрипник И. В. Методы исследования нелинейных эллиптических граничных задач. М.: Физматлит, 1990.

[6] Кондратьев В.А., Ландис Е. М. О качественных свойствах решений одного нелинейного уравнения второго порядка // Матем. сб. 1988. Т. 135. № 3. С. 346-360.

[7] Кондратьев В. А., Ландис Е. М. Полулинейные уравнения второго порядка с неотрицательной характеристической формой // Матем. заметки. 1988. Т. 44. № 4. С. 457-468.

[8] Туваев М. В. Об устранимых особых множествах решений квазилинейных эллиптических уравнений // Матем. сб. 1994. Т. 185. № 2. С. 107-114.

[9] Vazques J. L., Veron L. Removable Singularities of Strongly Nonlinear Elliptic Equations // Manuscripta Math. 1980/81. V. 33. P. 129-144.

[10] Vazques J. L., Veron L. Singularities of elliptic equations with an exponential nonlinearity // Math. Anal. 1984. V. 269. P. 119-135.

[11] Vazques J. L., Veron L. Isolated singularities of some semilinear elliptic equations // Journal of Diff. Equations. 1985. V. 60. P. 301-322.

[12] Littman W., Stampacchia G., Weinberger B. Regular Points for Elliptic Equations with Discontinuous Coefficients // Ann. Scuola Norm. Super. Pisa. Ser. 3. 1963. V. 17. № 1-2. P. 43-77. 